Kansas State University Libraries

New Prairie Press

\title{
APPROPRIATE STATISTICAL METHODS FOR COMPARING SOURCES OF NUTRITIONAL METHIONINE
}

D. D. Kratzer

R. C. Littell

Follow this and additional works at: https://newprairiepress.org/agstatconference

Part of the Agriculture Commons, and the Applied Statistics Commons

\section{(c) (1) $\Theta($}

This work is licensed under a Creative Commons Attribution-Noncommercial-No Derivative Works 4.0 License.

\section{Recommended Citation}

Kratzer, D. D. and Littell, R. C. (2006). "APPROPRIATE STATISTICAL METHODS FOR COMPARING SOURCES OF NUTRITIONAL METHIONINE," Conference on Applied Statistics in Agriculture.

https://doi.org/10.4148/2475-7772.1121

This is brought to you for free and open access by the Conferences at New Prairie Press. It has been accepted for inclusion in Conference on Applied Statistics in Agriculture by an authorized administrator of New Prairie Press. For more information, please contact cads@k-state.edu. 


\title{
APPROPRIATE STATISTICAL METHODS FOR COMPARING SOURCES OF NUTRITIONAL METHIONINE
}

\author{
D. D. Kratzer ${ }^{1}$ and R. C. Littell ${ }^{2}$ \\ ${ }^{1}$ E-Sci, DBA, Olivet, MI 49076 \\ ${ }^{2}$ Department of Statistics, University of Florida Gainesville 32611-0339;
}

\begin{abstract}
Kratzer and Ash(1996) presented Experimentation Science as a process to accomplish the Scientific Method with a complete protocol including relevant statistical design and analyses The first principal to sound Experimentation Science is the principle of Relevance. This is a case study primarily of Relevance in Experimentation Science. In our consulting work we found a so called "performance" design as not relevant because of the use of null hypothesis testing to promote a concept of equivalence. The best alternative involves equivalence testing, more replication and representative-ness. Secondly we found a dose response design for two products where non-linear asymptotic regression is misused in applying Bioassay techniques to estimate a single relative biological efficacy (RBV) because the basic assumption of sameness of mathematical form does not hold. We offer a relevant model which involves predicted differences in the relevant zone of commercial use (Vazquez- Añón, M et al, 2006b, GonzalesEsquerra et al, 2007).
\end{abstract}

\section{Introduction}

There are two primary product forms of supplemental L-methionine (L-met) activity commercially available for supplementation of Met deficient diets; DL-2-hydroxy-4-(methylthio) butanoic acid (HMTBa) most commonly available as an $88 \%$ solution with $12 \%$ water (ALIMET ${ }^{\circledR}$ feed supplement, registered trademark of Novus International, Inc, St. Louis, MO; Rhodimet AT-88®, registered trademark of Adisseo, Paris, France; Sumimet-L ${ }^{\circledR}$, registered trademark of Sumitomo Chemical, Tokoyo, Japan), and dry DL-methionine, (DLM, 99\% powder). While these compounds both provide L-met activity to avian and mammalian species alike, they are chemically different in that HMTBa has a hydroxyl group at the asymmetric carbon whereas DLM has an amino group. Clearly, HMTBa is not an amino acid as presented to the animal and not a nutrient, but a nutrient precursor. This results in a substantial number of metabolic differences between these products once they are made available for absorption in the gastrointestinal tract of the animal (Dibner, 2003, Lobley et al, 2005, Wester et al., 2005).

These compounds have been commercially available and used in animal production systems for over 50 years; however, there remains controversy and confusion with respect to the relative bioavailability value of HMTBa and DLM (i.e. bioavailability of a nutrient in relation to a response obtained with a standard reference material with known bioavailability or relative bioavailability value; RBV). This situation is fueled by continued publication of individual product comparisons in relatively small experiments as well as compilations i.e. "desk studies" of published results with apparently conflicting conclusions (Jansman et al. 2003; Vazquez-Anon et al, 2006a).

\section{Experimental Designs (65\% design)}


One experimental design called the "performance or 65\%" design has been used to compare responses to HMTBa in its commercial form and DLM on a product basis without accounting for the $12 \%$ water contained in the product. A variety of these types of comparisons between HMTBa and DLM have been previously described (Peak et al, 2002). It appears that this approach was initially based on the fact that HMTBa in commercial form contained 65\% to $69 \%$ HMTBa monomer, with $16 \%$ to $18 \%$ HMTBa dimer and 2 to 3\% HMTBa trimer while DLM is 99\% monomer. These ester linkages between HMTBa molecules are the result of reducing the water content to $12 \%$ in the commercial form of HMTBa liquid and like normal fatty acid esters, have been shown to be hydrolyzed in the intestine to monomer and available for use as a methionine source (Martin-Venegas et al, 2006).

Regardless of its origin, the 65\% design is constructed using a ratio of 65\% DLM (DLM65) to $100 \%$ of $88 \%$ HMTBa and 12\% water (ALIMET100). The stated objective is to demonstrate DLM65 is equivalent to ALIMET100. Diets are formulated with this assumption and if the results show no difference in ALIMET100 versus DLM65 then it is concluded that the product Alimet must be $65 \%$ of the product DLM. For example: Alimet is added at $0.326 \%$ of diet which results in $0.88 * 0.326 \%=0.286 \%$ added methionine for ALIMET100. DLM is added at $0.65 * 0.326=0.212 \%$ which results in $0.99 * 0.212 \%=0.21 \%$ added methionine for DLM65. Figure 1 shows where to expect the responses of these two groups in commercial broiler performance. There are two main talking points that can be addressed when faced with this trial. First the objective to demonstrate equivalence is not relevant in a test for difference. Second, in practical industry diets at one location with limited replication of experimental units, there is probably not enough power to show a statistically detectable difference for ALIMET100 versus formulating DLM65, especially if the Alimet100 group results in an over supplementation on the plateau. This bias may be further exaggerated if the dose response curves are more quadratic than asymptotic as suggested by Vasquez-Anon et al (2006) and ALIMET100 occurs on the downward portion of a quadratic response curve.

Statements from these 65\% designs such as "no significant difference $\mathrm{p}>.05$ ” provides no evidence for equivalence and this statement is easily misinterpreted as being the power of the test rather than the failure of the experiment to distinguish between the two groups. The Journal of Animal Science, Journal of Dairy Science (2006) Guidelines and the FDA Global Harmonization of Statistical Principles (1997) reiterate the fallacy of such statements. A clearer interpretation of the $65 \%$ experiment would be reflected by presenting confidence intervals on the differences or Least Significant Differences to indicate the lack of precision of such studies.

\section{Experimental Designs (Dose Response Designs)}

Another experimental design used to compare these two forms of MET is a factorial arrangement of 2 sources (Alimet and DLM) by $n$ doses. Usually $n$ equals 3 or more added levels starting with zero up to adequate nutritional levels. They usually have one or more nutritionally deficient, commercially irrelevant levels, which have been justified as necessary to show significant differences using a non-linear common plateau asymptotic regression (NLCPAR) model to compare the two Met sources i.e. they add irrelevant treatment groups to conform to a model. The longstanding controversy with respect to HMTBA and DLM efficacy is due at least in part to the misapplication of bioassay methodology for estimation of a single RBV with the NLCPAR model. The validity of approach is predicated on certain specific assumptions. In this case, 
Finney (1978) defined the technique as the comparison of a standard product ' $S$ ' to a test dilution ' $T$ '. The implicit assumption in nutritional studies is that the active compounds being compared are the same and that in the case of the two Met sources, DLM was considered the standard product ' $S$ ' and HMTBA the test dilution ' $T$ '. Littell et al. (1997) indicated that to be a valid comparison $S$ and $T$ must have the same mathematical form of dose response. Finney (1978) describes this further by stating that: "even a small discrepancy between the forms of the two curves would prove invalidity" and "in particular, if the S response curve asymptotically approaches a limiting value, that T must have the same asymptote”. In other words, for a valid comparison of HMTBA and DLM using Finney’s relative potency methodology, one product must function as a dilution of the other. That is, there is a dilution factor $\mathrm{k}$ such that the mean response to a dose ' $x$ ' of the standard product is the same as the mean response to a dose ' $k x$ ' of the test product. The value of $\mathrm{k}$ is the potency of the test product relative to the standard product. More technically, if $\mathrm{f}(\mathrm{x})$ and $\mathrm{g}(\mathrm{x})$ are the response curves for products $\mathrm{S}$ and $\mathrm{T}$, respectively, then for any dose $\mathrm{x}, \mathrm{f}(\mathrm{x})=\mathrm{g}(\mathrm{kx})$. This condition is impossible unless the two response curves have the same asymptote.

Before properly applying the NLCPAR bioassay method, some effort must be made to test the validity of the model's basic assumptions. It appears that such tests of assumptions have not routinely been examined. A recently prepared meta-analysis sought to resolve the Met source RBV controversy for poultry and swine and employed the NLCPAR methodology as a primary means of evaluating published Met source comparisons (Jansman et al, 2003). Indeed, no reference was made to the basic assumptions of the model when the authors reported relative HMTBA:DLM efficacy estimates of 77\%, 82\% 83\% and 83-101\% for broilers, pigs, layers and turkeys respectively. These single values were based on averages across several studies to which the authors applied either NLCPAR models or linear models depending on set criterion. The report indicated that of the approximately 132 broiler citations surveyed (representing the majority of published data comparing these two compounds) 17 experiments fit the selection criteria for inclusion in the compilation with respect to BWG response to the two Met sources. Of those 17 citations, four studies were not appropriate for nonlinear analyses since the response curves showed no curvature, probably due to the low levels of supplementation used in those experimental designs. The remaining 13 broiler studies with curvature were used to evaluate validity of the basic assumptions required for application of the NLCPAR method. Each of these experimental designs had at least three non-zero supplemented doses of the Met sources and an un-supplemented control and all had suboptimal doses of both HMTBA and DLM. In order to test the assumptions of same dose and plateau responses, the treatment means for the 13 data sets representing curvilinear BWG responses were used to estimate equations in the form of nonlinear separate plateau asymptotic regression (NLSPAR) predicting the plateau response for each Met source separately instead of a common plateau NLCPAR. The following model in the SAS Procedure NLMIXED (SAS Institute, 2003), was used to simultaneously fit separate curves for broiler BWG for each product:

$$
\text { BWG }=\text { b1 }+ \text { D*(b2 *(1-exp }(\mathrm{b} 3 * \text { DOSEDLM })))+H^{*}(\mathrm{~b} 4 *(1-\exp (\mathrm{b} 5 * \text { DOSEHMB }))) \text {; }
$$

$\mathrm{D}$ and $\mathrm{H}$ were columns of $0=$ no or $1=$ yes to indicate which product is involved in the response, and DOSEDLM and DOSEHMB are the levels of Met for each product DLM or HMTBA respectively. 
The results in Table 1 show the estimated plateaus for HMTBA and DLM along with sign of the difference between the two plateaus. To test the hypothesis that HMTBA and DLM have equal plateaus across all studies, a non-parametric binomial test, i.e. a sign test, was used to test the hypothesis of equal plateaus, (Conover, 1980). The sign test assumes that if the two sources have equal plateaus a random sample of studies will yield a distribution of + and - differences which follow a binomial distribution with a frequency of 50\%. That is a 50:50 distribution of + and - is expected if the plateaus are truly the same. For 11 out of 13 of these studies the plateau difference (HMTBA-DLM) was positive, meaning the predicted HMTBA plateau was greater than the DLM plateau. This is a very unusual result if the plateaus were in fact equal. The chance of such is $\mathrm{p}<0.01$. Therefore, based on the sign test, the hypothesis of equal plateaus for HMTBA and DLM was rejected $(\mathrm{p}<0.01)$. It is now reasonable to conclude the following: DLM and HMTBA do not have the same form of dose response, and HMTBA is not a dilution of DLM. These results also demonstrate that application of the NLCPAR method was not an appropriate analysis for the referenced meta-analysis (Jansman et al, 2003) and that the conclusions regarding RBV of HMTBA and DLM from it and other such comparisons (Potter et al, 1984) are, therefore, suspect. Most importantly, these results indicate there is not a single RBV value for HMTBA and DLM, but instead their relative efficacies vary with dose.

\section{Dose Response Combined with 65\% Design}

Figure 2 (Kim et al. 2006. J. Anim. Sci.84:104-111) is an example of a combination of the 65\% design and a dose response. This study was with pigs and the response variable was nitrogen balance which is a surrogate variable for growth. The main relevance issue here is that the design is in the irrelevant region of practical supplementation. The design is used to apply bioassay logic of slope ratio to estimate a single RBV in the irrelevant zone. For any practical application it would have to be assumed that the RBV estimated in the irrelevant zone applies to the relevant zone of supplementation. Testing only in the linear phase of response is questionable since methionine response is known to reach a plateau. And as we have shown in Table 1 the response curves for these two products in poultry have different plateaus and that a single RBV is not appropriate for the entire range. Nutritionists often use poultry to model responses in swine so these results are suspect until the assumptions of bioassay are found to be appropriate for testing methionine sources for swine.

\section{Impact of misuse of bioassay technique}

To illustrate the impact of the assumption of common plateaus for HMTBA and DLM on determination of RBV estimates, a single intermediate set of data from the referenced compilation was chosen (Schutte and de Jong, 1996). Figure 3 shows the observed means for each product with the level of Met supplementation on the x-axis and 28-day BWG on the y-axis with 4 levels of equimolar supplementation for both HMTBA and DLM over a common unsupplemented basal. Most such studies use the so called ANOVA approach for the first or only analysis. The so called ANOVA approach involves all pair wise comparisons to try to make decisions. That ANOVA procedure is not a relevant analysis of an experiment which was designed to compare the trends of two different products. The subsequent irrelevant results create confusion since they provide little if any information about the interrelationships of the designed treatment groups. The conclusions by the authors was that both sources of methionine resulted in significant increases in 28 day body weight over the un-supplemented control. "Analysis of variance indicates that there was no significant difference between bodyweights for HMTBA or 
DLM supplemented birds, however, their gain was numerically lower for HMTBA at the 2 lowest levels and numerically higher at the two highest levels.” In addition to the ANOVA analyses Schutte and de Jong applied the NLCPAR model in the bioassay approach to estimate a single RBV of .89.

Table 2 shows the SAS program with PROC NLMIXED code to fit the same NLCPAR model as in Schutte and de Jong (1996).

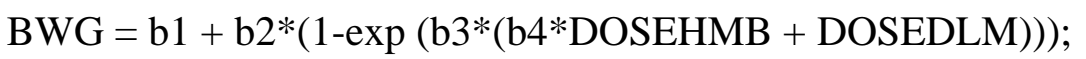

Figure 4 shows the Schutte and de Jong (1996) data with the fitted NLCPAR curves. This exponential model has three main properties: first, it has a separate curved line both for DLM and HMTBA; second, the two curved lines start at the same point (b1); and third, the two curved lines end at the same point, at a common plateau (b1 + b2) estimated to be $1714 \mathrm{~g}$. Due to the assumption of a common starting point and a common ending point, DLM and HMTBA differ in only one respect, i.e., the rate at which each curve approaches the assumed common plateau. In the formula for the NLCPAR model above, the rate of change in BWG as the level of DLM increases is estimated by the coefficient b3. The ratio of change in BWG for HMTBA, relative to DLM, is estimated by b4, i.e. RBV. Using NLCPAR, b4 estimates that a single ratio of 0.89 exists for HMTBA, relative to DLM over the entire range of supplementation.

The conclusion from Table 1 dictates the use of non-linear separate plateau asymptotic regression (NLSPAR) models for these data. Table 3 shows the SAS code in PROC NLMIXED used to fit the following separate plateau NLSPAR equations:

BWG = b1 + D*(b2(1-exp(b3*DOSEDLM $)))+\mathrm{H}^{*}(\mathrm{~b} 4(-\exp (\mathrm{b} 5 * \mathrm{DOSEHMB})))$;

Figure 5 shows the Schutte and de Jong (1996) data with the fitted NLSPAR curves. It is apparent by visually comparing Figure 4 versus Figure 5 that separate dose response curves gives a better fit to the data; however, a method to objectively determine goodness of fit is needed. The usual $\mathrm{R}^{2}$ criterion for assessing goodness of fit of regression models is designed for linear models and has technical complications when used with non-linear models. Other criteria, such as the Bayesian Information Criterion (BIC), are more appropriate, and are based on the statistical principle of likelihood (Sy and Gupta, 2004) and can also be used to test polynomial models such as quadratics. PROC NLMIXED computes BIC and several other goodness of fit statistics. The BIC value for the NLCPAR model shown in Table 2 was 84.5. The BIC value for the NLSPAR model shown in Table 3 was 74.9. Since smaller BIC values indicate better fit, the separate plateau model fits these data significantly better than the common plateau model.

Another useful feature of PROC NLMIXED is the ESTIMATE statement that allows estimates and tests of significance of functions of the parameter estimates. Table 3 shows an ESTIMATE statement that tests the significance of the difference between predicted plateaus (b4 - b2). For these data the HMTBA had a significantly higher plateau than DLM $(\mathrm{p}<.0024$. Due to the relatively low power of such designs an inability to determine a significant difference between plateaus in every case is not surprising. However, the combination of a group of studies such as 
in Table 1 provides a broad set of experiments that clearly identifies the separate plateau relationship of HMTBA and DLM.

Results in Table 1 provide strong evidence that the response curves for HMTBA and DLM have different plateaus; thus, a single value for RBV is not appropriate for the entire Met supplementation dose range. Consequently, the two products should not be compared using Finney's (1978) relative potency method. More appropriate methods are needed for unbiased comparison of the products across the entire dose range. Here again we can use the ESTIMATE statement in NLMIXED where we fit separate curves. Table 4 shows the ESTIMATE statements used to obtain predicted differences and $95 \%$ confidence limits (CL) on the predicted difference at selected levels of met supplementation.

Figure 6 shows a graphical presentation of the selected predicted differences and their 95\% CL. Where the upper limit is less than zero, the DLM BWG response is significantly greater than HMTBA i.e. when Met supplementation levels are from 0.04 to $0.09 \%$. Where the lower limit is greater than zero HMTBA is significantly greater than DLM, i.e. Met supplementation levels are between 0.15 to $0.22 \%$ and where the limits include zero, HMTBA and DLM are not significantly different. This result has often been misrepresented as proof of equality or equivalence. This is a misuse of null hypothesis testing. Not significantly different does not equate to no difference. "Not significantly different' is more appropriately interpreted as "not distinguished by this experiment”. The question then is: How much difference could have been detected? We recommend authors present Least Significant Differences or confidence limits on these important tests so the reader can see what magnitudes of differences that may have been missed due to lack of experimentation power

Another consideration for model fitting in this area is that other classes of models may fit better and or have a more relevant biological meaning. In experiments with broilers and turkeys, best fit equations for HMTBa were found to be linear while quadratic models better described the DLM response (Vazquez- Añón, M et al, 2006b, Gonzales-Esquerra et al, 2007), while in young pigs linear equations were best fit models for both products (Yi et al, 2006). Vazquez-Anon et al (2006a) found that in 100 experiments in a literature review of all available broiler studies in which HMTBa and DLM were both used, that weight gain response showed separate quadratic models for these two products. The BIC evaluation in PROC NLMIXED can be used to evaluate the best fitting models and then predict the differences and confidence intervals as done here with the asymptotic models.

Since we have established mathematical models for the prediction equation we can now get a continuum of predicted differences using ESTIMATE statements to predict differences at use levels not included in the study. Since these models use all the data to determine differences at individual points, in general they will be more powerful than pair-wise comparisons of the analysis of variance procedure. Finally, the separate plateau modeling provides an appropriate means of determining which product to use based on where in the Met response curve the nutritionist decides to feed.

\section{Summary}

The 65\% "performance” design is a misuse of null hypothesis testing. The best alternative involves equivalence testing. At the very least, the reporting of results from such experiments 
should present confidence intervals and or LSDs to indicate the sensitivity of the tests. The nonlinear common plateau regression model has been used in a Bioassay framework to estimate an RBV of HMTBA and DLM in a variety of species. Inherent in this procedure is the assumption that HMTBA is a dilution of DLM and would then be expected to follow the same form of dose response. However, until now, there has been no statistical discussion regarding the appropriateness of this model for such comparisons. Examination of the model's assumptions using a set of 13 comparisons in broilers (Jansman et al., 2003) and testing for validity has yielded the conclusion that application of the NLCPAR methodology for determining RBV of HMTBA and DLM is inappropriate and as such leads to erroneous conclusions regarding the RBV of the two Met sources. The evidence presented here indicates separate plateau models should be used when comparing these two products. These more relevant models can then be used for predictions of differences between HMTBA and DLM at levels of expected use.

\section{References}

Conover. W. J. 1980. Practical nonparametric statistics. 2nd ed: John Wiley \& Sons, New York, NY.

Dibner, J.J. ,2003. Review of the metabolism of DL-2-hydroxy-4-methylthio-butanoic acid. World Poultry Sci. J. 59:99-110.

De Groote, G., G. Huygebaert, D. C. Bruyer, and M. Vanbelle., 1990. Efficacy of the dimeric form of methionine hydroxy analog free acid as determined by chick bioassay. Report and Ann. Zootech. 39:45-51.

Gonzales-Esquerra, R. M. Vázquez- Añón, T. Hampton, T. York, S. Feine, C. Wuelling, and C.D. Knight. 2007. Evidence of a different dose response in turkeys when fed 2-hydroxy4(methylthio) butanoic acid versus DL-methionine. Poult. Sci. 86:517-524.

Esteve-Garcia E., and L. L. Llaurado. 1997. Performance, breast meat yield and abdominal fat deposition of male broiler chicken fed diets supplemented with DL-Methionine and DLMethionine hydroxy analogue free acid. Br. Poult. Sci. 38:397-404.

Finney, D.J. 1978. Statistical Method in Biological Assay. ( 3rd Edition) Griffin, London.

Jansman, A. J. M., J. de Jong, and G. M Beelen. 1998. Biological effectiveness of different methionine sources in broilers. TNO report number 98-31126.

Jansman, A. J. M., C. A. Kan and J. Wiebenga. 2003. Comparison of the biological efficacy of DL-methionine and hydroxyl-4-methylthiobutanoic acid (HMB) in pigs and poultry. IDLelystad No. 2209.

Journal of Animal Science. 2006. http://www.asas.org.

Journal of Dairy Science. 2006. 89:371-381. or http://www.jds.org.

Kratzer D.D..and K.A.Ash. 1996. Experimentation Science: A process approach for the complete design of an experiment. Proceedings of the 1996 Kansas State University Conference on Applied Statistics in Agriculture

Kratzer, D. D. and R. C. Littell. 2006. Appropriate statistical methods to compare dose responses of methionine sources. Poultry Sci. 85:947-954.

Kim, B. G., M. D. Lindemann, G. L. Cromwell, and M. Rademacher. 2005. Efficacy of liquid and DL-methionine hydroxyl analog free acid and DL-methionine as methionine sources for pigs. J. Anim. Sci.84:104-111.

Lemme, A., D. Hoehler, J. J. Brennan, and R. F. Mannion. 2002. Relative effectiveness of methionine hydroxyl anlog compared to DL-methionine in broiler chickens. Poult. Sci. 81:838-45. 
Littell, R.A. , P. R. Henry, A. J. Lewis and C. B. Ammerman. 1997. Estimation of relative bioavailability of nutrients using SAS procedures. J. Anim. Sci. 75:2672-2683.

Lobley, G.E., T. J. Wester, A. G. Calder, D. S. Parker, J. J. Dibner, and M. Vázquez-Añón. 2006. Absorption of 2-hydroxy-4-methylthiobutyrate (HMTBA) and Conversion to Met in Lambs. J. Dairy Sci. 89: 1072-1080.

Martin-Venegas, R, G. F. Soriano-Garcia, M. P. Vinardell, P. A. Geraert, and R. Ferrer. 2006. Oligomers are not the limiting factor in absorption of DL-2-hydroxy-4-(methylthio) butanoic acid in the chicken small intestine. Poult. Sci. 85:56-63.

Peak S. D. , T. J. Walsh, and J.J. Dibner. 2002. Trial design important for comparing feed ingredients. Feedstuffs Vol 74, No. 41. October 7, 2002.

SAS Institute. 2003. SAS User’s Guide: Statistics. Version 9.0. SAS Institute, Inc., Cary, NC.

Schutte, J. B., and J. de Jong. 1996. Biological efficacy of DL-methionine hydroxy ananlog free acid compared to DL-methionine in broiler chicks as determined by performance and breast meat yield. Agribiol. Res. 49(1):74-82.

Summers, J. D., S. Blackman, and S. Lesson. 1987. Assay estimating the potency of various methionine-active sources. Poult. Sci. 66:1779-1787.

Sy, B. K., and A. K. Gupta. 2004. Information-statistical data mining: warehouse integration with examples of oracle basics. Page 80 in Akaike and Schwarz Information Criteria. Kluwer Academic Publishers. Massachusetts, USA.

Thomas, O. P., E. H. Bossard, S. D. Crissey, and K. P. Engler. 1984. Further studies on the evaluation of DL-methionine and related compounds. Page 41-45 in Proceedings of Maryland Nutrition Conference for Feed Manufactures.

Thomas, O. P., C. Tamplin, S. D. Crissey, E. Bossard, and A. Zuckerman. 1991. An evaluation of methionine hydroxyl analog free acid using a nonlinear (exponential) bioassay. Poult. Sci. 70:605-610.

Uzu, G..1987. Nutritional efficacy of DL-methionine and DL-methionine hydroxy analogue acid in corn soybean meal lupin diet. Proceedings for the European Poultry Conference (August, 1986). Pp 612-613.

Van Weerden E J., J. B. Schutte, and H. L Bertram. 1992. Utilization of polymers of methionine hydroxyl analogue free acid (MHA-FA) in broiler chicks. Arch. Geflugelk. 56 (2): 63-68.

Wester, T.J., M. Vázquez-Añón, J. Dibner, D. S. Parker, A. G. Calder, and G. E. Lobley. 2006.

Hepatic Metabolism of 2-hydroxy-4-thiomethylbutyrate (HMTBA) in Growing Lambs. J. Dairy Sci. 1062-1071.

Vazquez- Añón, M., M. Mehmeyer, C. W. Wuelling, T. Hampton, C. D. Knight, and J. J. Dibner, 2003. Differential response to 2-hydroxy-4-methylthio-butanoic acid and DL-methionine above requirement on broilers and pig performance and iron metabolism. Pages 725-729 in Progress in Research on Energy and Protein Metabolism. EAAP publication No 109. Rostock-Warnemunde, Germany.

Vázquez-Añón, M., D. Kratzer, R. Gonzalez-Esquerra, G. F. Yi, and C.D. Knight. 2006a. A multiple regression model approach to contrast the performance of 2-hydrixy-4-methylthio butanoic acid and DL-methonine supplementation tested in broiler experiments and reported in the literature. Poultry Sci. 85: 693-705.

Vázquez-Añón, M., R. Gonzalez-Esquerra, E. Saleh, T. Hampton, S. Ricther, J. Firman, and C. D. Knight. 2006b. Evidence for 2-Hydroxy-4-Methylthio Butanoic Acid and DLmethionine having a Different Dose-Response in Growing Broilers. Poult. Sci. 85:14091420. 
Table 1 Estimation of the plateau responses of 13 studies (Jansman et al, 2003) for body weight gain (BWG) of broilers fed 2-hydroxy-4-methylthio butanoic acid (HMTBA) and DL-methionine (DLM) using non-linear separate plateau asymptotic regression (NLSPAR)

*

\begin{tabular}{|l|l|l|l|l|}
\hline $\begin{array}{l}\text { Broiler } \\
\text { Study \# }\end{array}$ & Reference & $\begin{array}{l}\text { HMTBA BWG } \\
\text { Plateau }\end{array}$ & $\begin{array}{l}\text { DLM BWG } \\
\text { Plateau }\end{array}$ & $\begin{array}{l}\text { Sign of } \\
\text { Difference } \\
\text { HMTBA-DLM }\end{array}$ \\
\hline 2 & Jansman et al, 1998 & 1712.8 & 1809.3 & - \\
\hline 9 & Lemme et al, 2001 & 2630.2 & 2435.2 & + \\
\hline 10 & Lemme et al, 2001 & 2105.4 & 2042.2 & + \\
\hline 21 & $\begin{array}{l}\text { Schutte and de Jong, } \\
1996\end{array}$ & 1753.8 & 1689.2 & + \\
\hline 26 & $\begin{array}{l}\text { Esteve-Garcia \& } \\
\text { Laurado, 1999 }\end{array}$ & 59.97 & 58.53 & + \\
\hline 29 & De Groote et al., 1985 & 1963.1 & 1954.4 & + \\
\hline 54 & Uzu, G, 1987 & 1468.9 & 1458.4 & + \\
\hline 95 & Thomas et al., 1991 & 511.9 & 510.5 & + \\
\hline 96 & Thomas et al., 1991 & 527.3 & 513.5 & + \\
\hline 109 & Thomas et al., 1984 & 470.4 & 447.7 & + \\
\hline 110 & Van Weerden et al., 1992 & 728.7 & 683.5 & + \\
\hline 111 & Van Weerden et al., 1992 & 578.3 & 707.7 & - \\
\hline 116 & Summers et al., 1987 & 397.3 & 390.2 & + \\
\hline
\end{tabular}

- Sign test (Conover, 1980) rejects the hypothesis of equal plateaus for HMTBA and DLM $(\mathrm{P}<.01)$.

Table 2. PROC NLMIXED SAS code used to generate non-linear common plateau asymptotic regression (NLCPAR) equations for body weight gain (BWG) of 2-hydroxy-4methylthio butanoic acid (HMTBA) and DL-methionine (DLM) supplemented chickens (Schutte and de Jong, 1996).

data in;

input DoseDLM DoseHMTBA D H bwg;

cards;

$\begin{array}{lllll}0 & 0 & 0 & 0 & 1453 \\ 0.04 & 0 & 1 & 0 & 1593 \\ 0.09 & 0 & 1 & 0 & 1660 \\ 0.15 & 0 & 1 & 0 & 1666 \\ 0.22 & 0 & 1 & 0 & 1698 \\ 0 & 0.04 & 0 & 1 & 1561 \\ 0 & 0.09 & 0 & 1 & 1633 \\ 0 & 0.15 & 0 & 1 & 1704 \\ 0 & 0.22 & 0 & 1 & 1722\end{array}$

;

title1 'Asymptotic regression models'; 


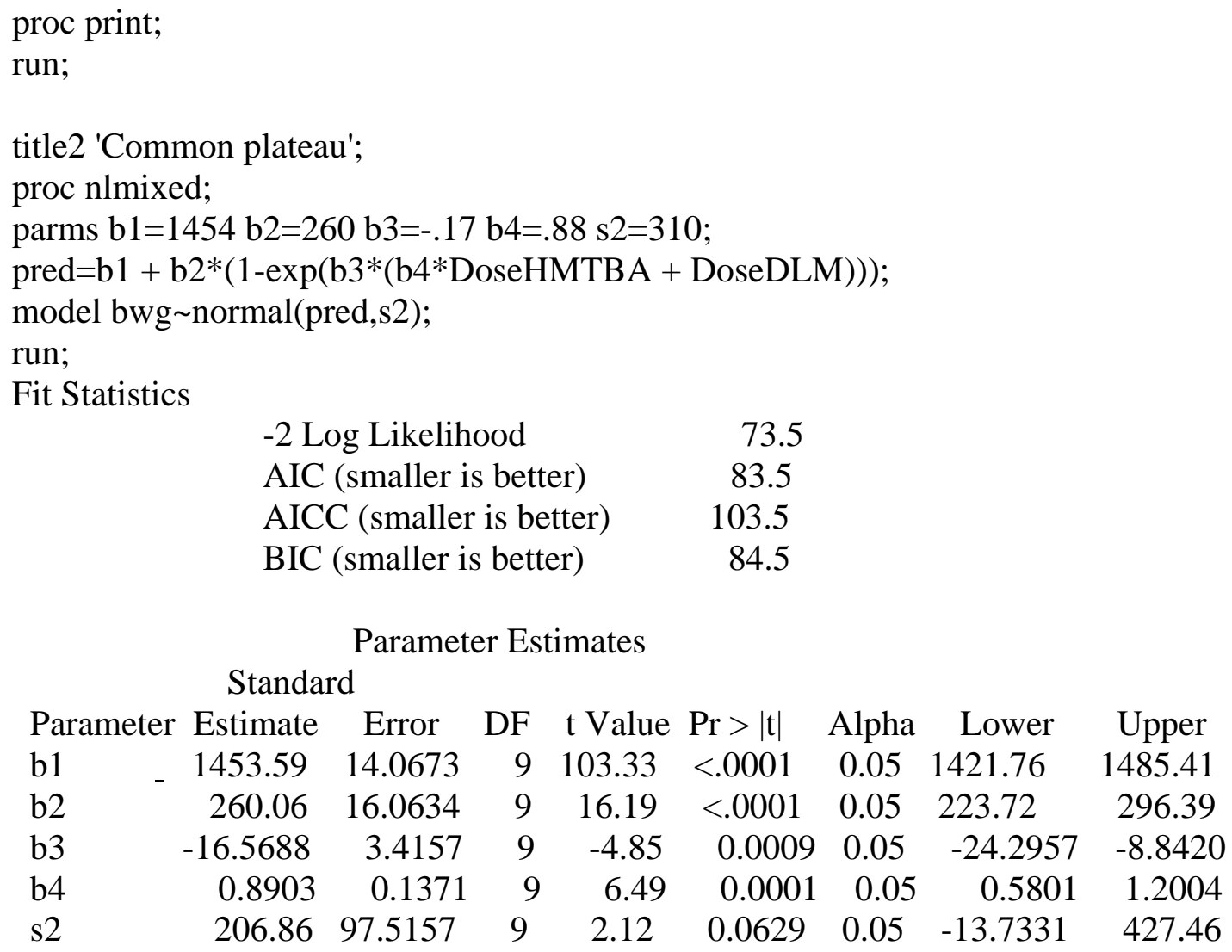

Table 3. PROC NLMIXED SAS code used to generate non-linear separate plateau asymptotic regression (NLSPAR) equations for body weight gain (BWG) of 2-hydroxy-4methylthio butanoic acid (HMTBA) and DL-methionine (DLM) supplemented chickens (Schutte and de Jong, 1996).

data in;

input DoseDLM DoseHMTBA D H bwg; cards;

$\begin{array}{lllll}0 & 0 & 0 & 0 & 1453 \\ 0.04 & 0 & 1 & 0 & 1593 \\ 0.09 & 0 & 1 & 0 & 1660 \\ 0.15 & 0 & 1 & 0 & 1666 \\ 0.22 & 0 & 1 & 0 & 1698 \\ 0 & 0.04 & 0 & 1 & 1561 \\ 0 & 0.09 & 0 & 1 & 1633 \\ 0 & 0.15 & 0 & 1 & 1704 \\ 0 & 0.22 & 0 & 1 & 1722\end{array}$

;

title1 'Asymptotic regression models';

proc print;

run;

title2 'Separate plateaus'; 
proc nlmixed;

parms b1=1454 b2=260 b3=-.2 b4=300 b5=-.2 s2=310;

pred=b1 + D*(b2*(1-exp(b3*DoseDLM $)))+\mathrm{H}^{*}(\mathrm{~b} 4 *(1-\exp (\mathrm{b} 5 *$ DoseHMTBA $)))$;

model bwg normal(pred,s2);

estimate 'PLATEAU DIFF' b4-b2;

Fit Statistics

-2 Log Likelihood

61.7

AIC (smaller is better) $\quad 73.7$

AICC (smaller is better) $\quad 115.7$

BIC (smaller is better) $\quad 74.9$

Parameter Estimates

Standard

Parameter Estimate Error DF $t$ Value $\operatorname{Pr}>|t|$ Alpha Lower Upper

$\begin{array}{lllllllll}\text { b1 } & 1453.60 & 7.1772 & 9 & 202.53 & <.0001 & 0.05 & 1437.36 & 1469.84\end{array}$

$\begin{array}{llllllllll}\text { b2 } & 235.65 & 9.1583 & 9 & 25.73 & <.0001 & 0.05 & 214.93 & 256.37\end{array}$

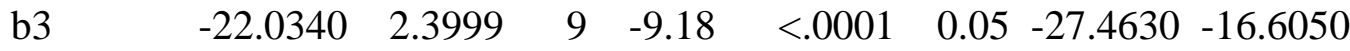

$\begin{array}{lllllllll}\text { b4 } & 300.50 & 14.0979 & 9 & 21.32 & <.0001 & 0.05 & 268.61 & 332.40\end{array}$

$\begin{array}{llllllllll}\text { b5 } & -10.7912 & 1.2826 & 9 & -8.41 & <.0001 & 0.05 & -13.6927 & -7.8898\end{array}$

Additional Estimates

Standard

Label Estimate Error DF t Value $\operatorname{Pr}>|\mathrm{t}|$ Alpha Lower Upper

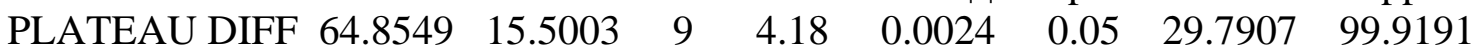

Table 4. PROC NLMIXED SAS code used to generate differences in predicted body weight gain (BWG) of DL-methionine (DLM) (Schutte and de Jong, 1996).

data in;

input DoseDLM DoseHMTBA D H bwg;

cards;

$\begin{array}{lllll}0 & 0 & 0 & 0 & 1453\end{array}$

$\begin{array}{lllll}0.04 & 0 & 1 & 0 & 1593\end{array}$

$\begin{array}{lllll}0.09 & 0 & 1 & 0 & 1660\end{array}$

$\begin{array}{lllll}0.15 & 0 & 1 & 0 & 1666\end{array}$

$\begin{array}{lllll}0.22 & 0 & 1 & 0 & 1698\end{array}$

$\begin{array}{lllll}0 & 0.04 & 0 & 1 & 1561\end{array}$

$\begin{array}{lllll}0 & 0.09 & 0 & 1 & 1633\end{array}$

$\begin{array}{lllll}0 & 0.15 & 0 & 1 & 1704\end{array}$

$\begin{array}{lllll}0 & 0.22 & 0 & 1 & 1722\end{array}$

;

title2 'Separate plateaus'; 
proc nlmixed;

parms b1 $=1454 \mathrm{~b} 2=260 \mathrm{~b} 3=-.2 \mathrm{~b} 4=300 \mathrm{~b} 5=-.2 \mathrm{~s} 2=310$;

pred=b1 + D*(b2*(1-exp(b3*DoseDLM)) + H*(b4*(1-exp(b5*DoseHMTBA)));

model bwg normal(pred,s2);

estimate 'PLATEAU DIFF' b4-b2;

estimate '.04' (b4*(1-exp(b5*.04))) - (b2*(1-exp(b3*.04)));

estimate '.06' (b4*(1-exp(b5*.06))) - (b2*(1-exp(b3*.06)));

estimate '.08' (b4*(1-exp(b5*.08))) - (b2*(1-exp(b3*.08)));

estimate '.09' (b4*(1-exp(b5*.09))) - (b2*(1-exp(b3*.09)));

estimate '.10' (b4*(1-exp(b5*.10))) - (b2*(1-exp(b3*.10)));

estimate '.12' (b4*(1-exp(b5*.12))) - (b2*(1-exp(b3*.12)));

estimate '.14' (b4*(1-exp(b5*.14))) - (b2*(1-exp(b3*.14)));

estimate '.155' (b4*(1-exp(b5*.155))) - (b2*(1-exp(b3*.155)));

estimate '.16' (b4*(1-exp(b5*.16))) - (b2*(1-exp(b3*.16)));

estimate '.18' (b4*(1-exp(b5*.18))) - (b2*(1-exp(b3*.18)));

estimate '.20' (b4*(1-exp(b5*.20))) - (b2*(1-exp(b3*.20)));

estimate '.22' (b4*(1-exp(b5*.22))) - (b2*(1-exp(b3*.22)));

run;

Figure 1: Anticipated response of DLM65 versus ALIMET100

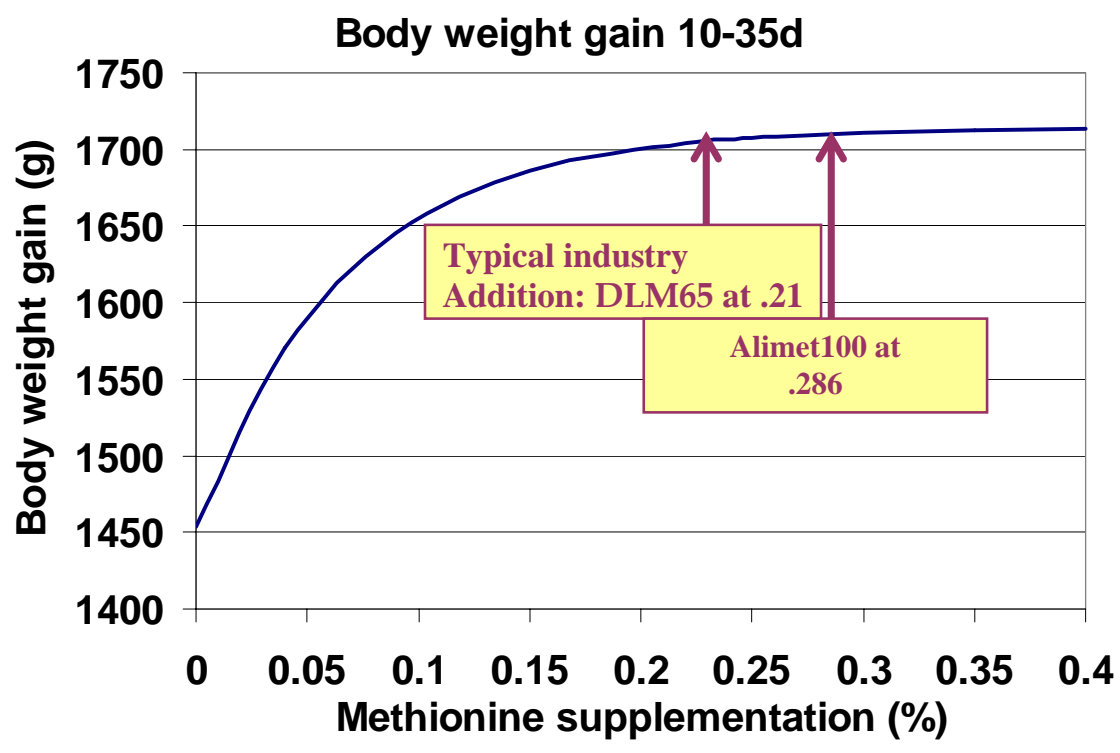


Figure 2. Example of a combination of the $65 \%$ design and slope-ratio in the irrelevant portion of the curve. (Kim et al, 2005).

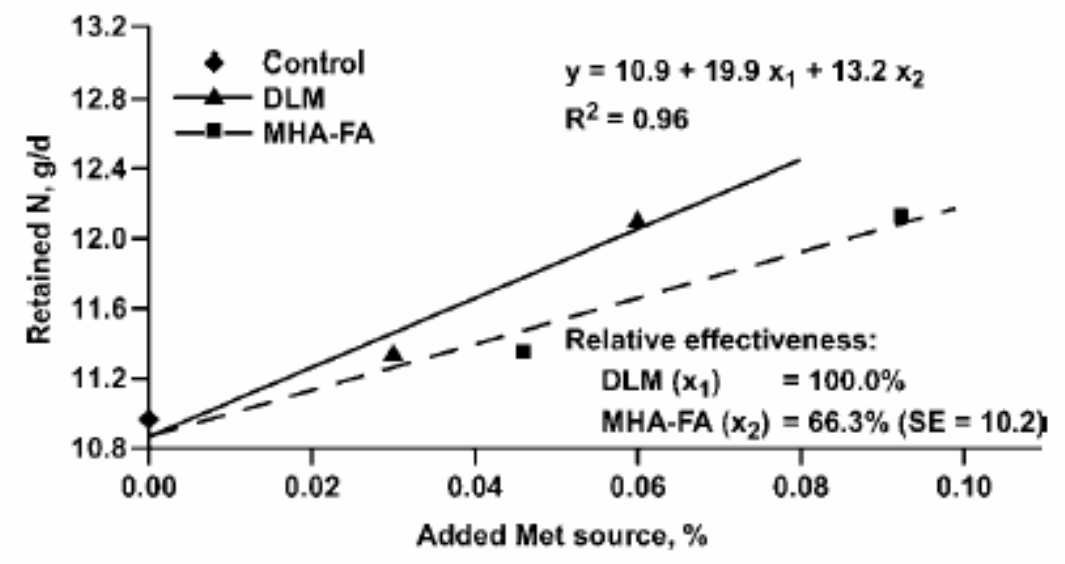

Figure 3: Mean values of 28-day body weight gains (g) in broiler chickens as reported by Schutte and de Jong (1996) using the ANOVA method with all possible pair wise comparisons.

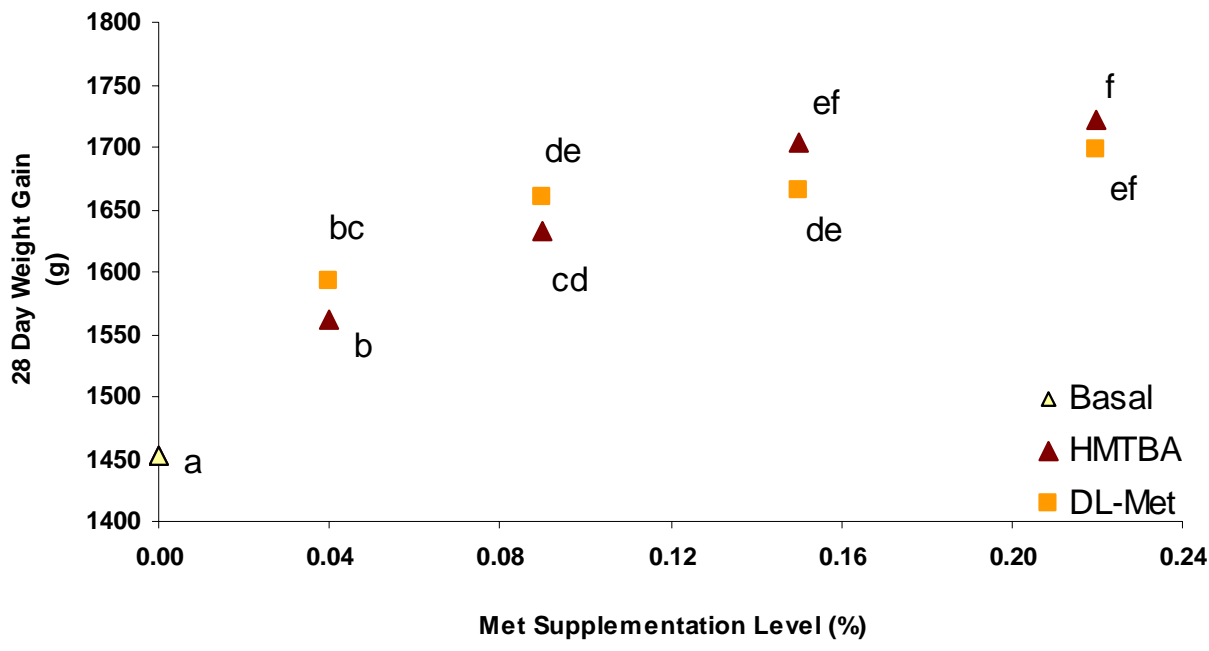


Figure 4: Estimates from fitting Non-linear separate plateau asymptotic regression

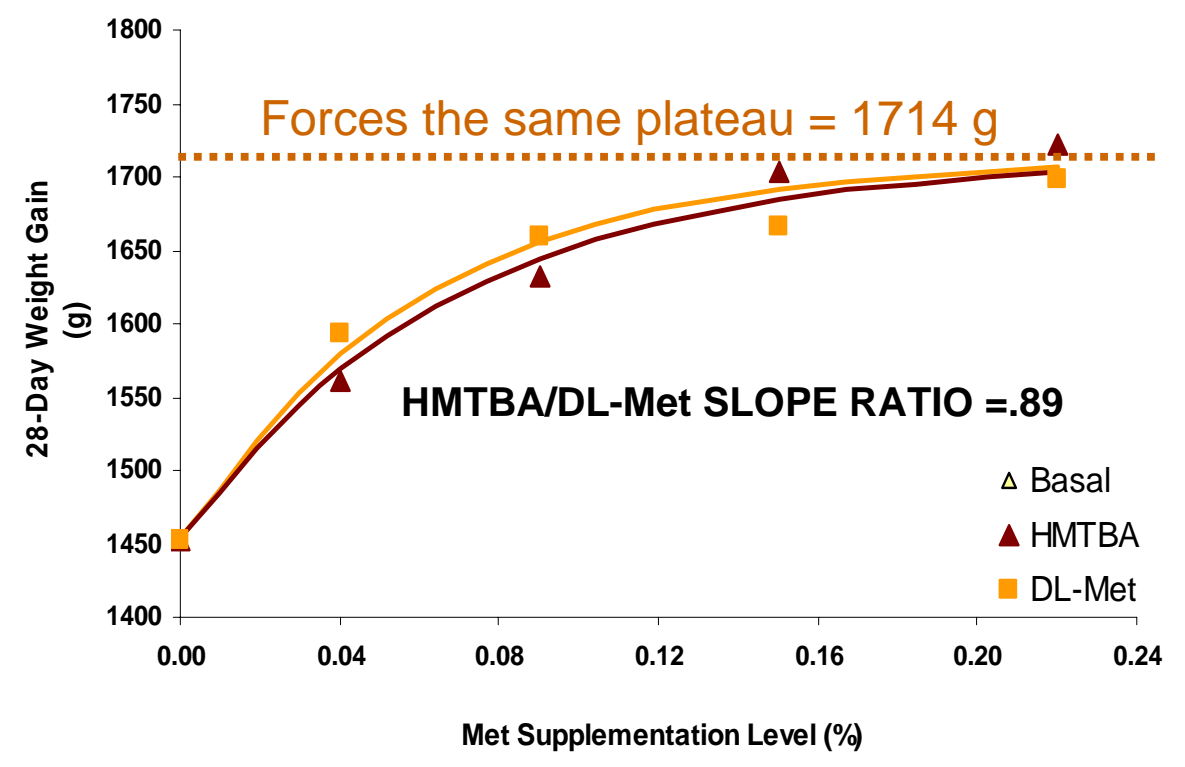

Figure 5. NLSPAR FIT

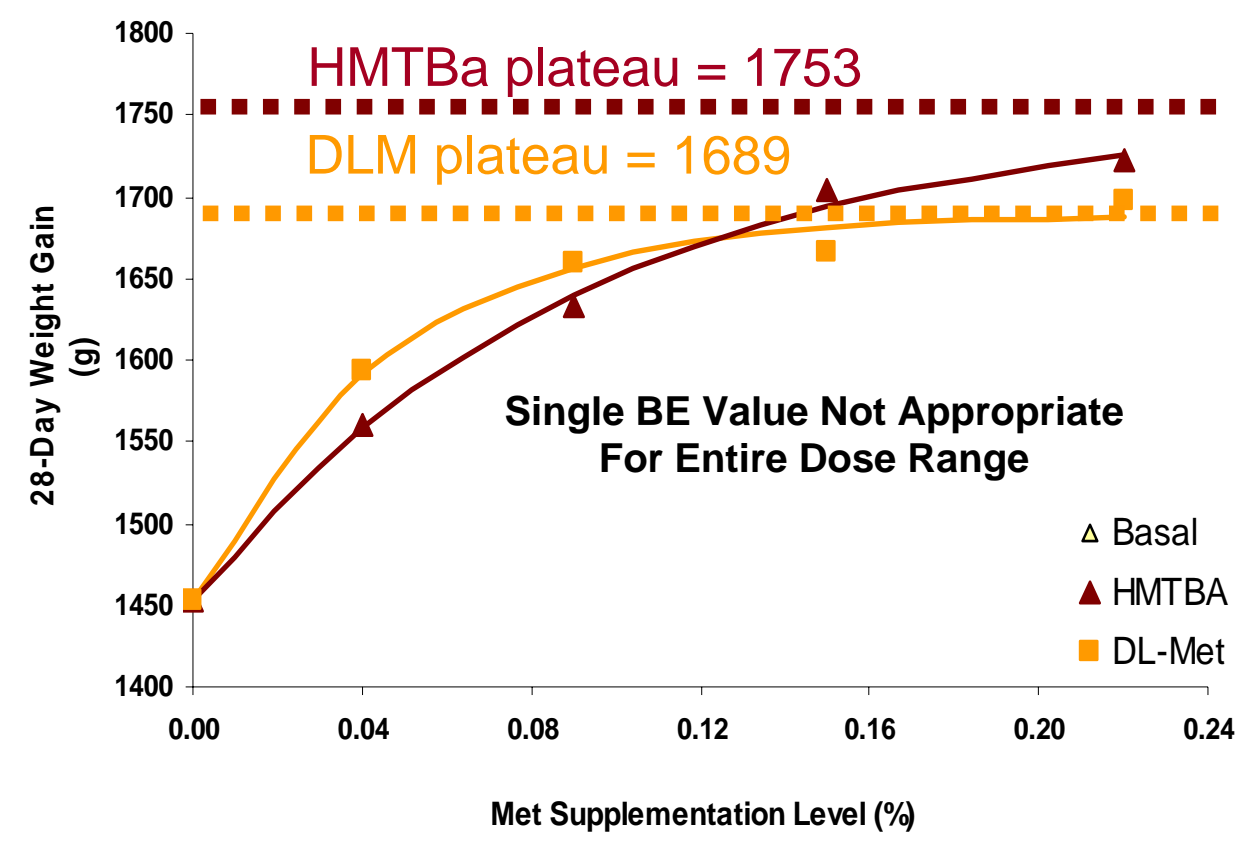


Figure 6. Predicted Differences with Associated Confidence Intervals Showing both Level of Supplementation and Total Methionine plus Cysteine on the X Axis.

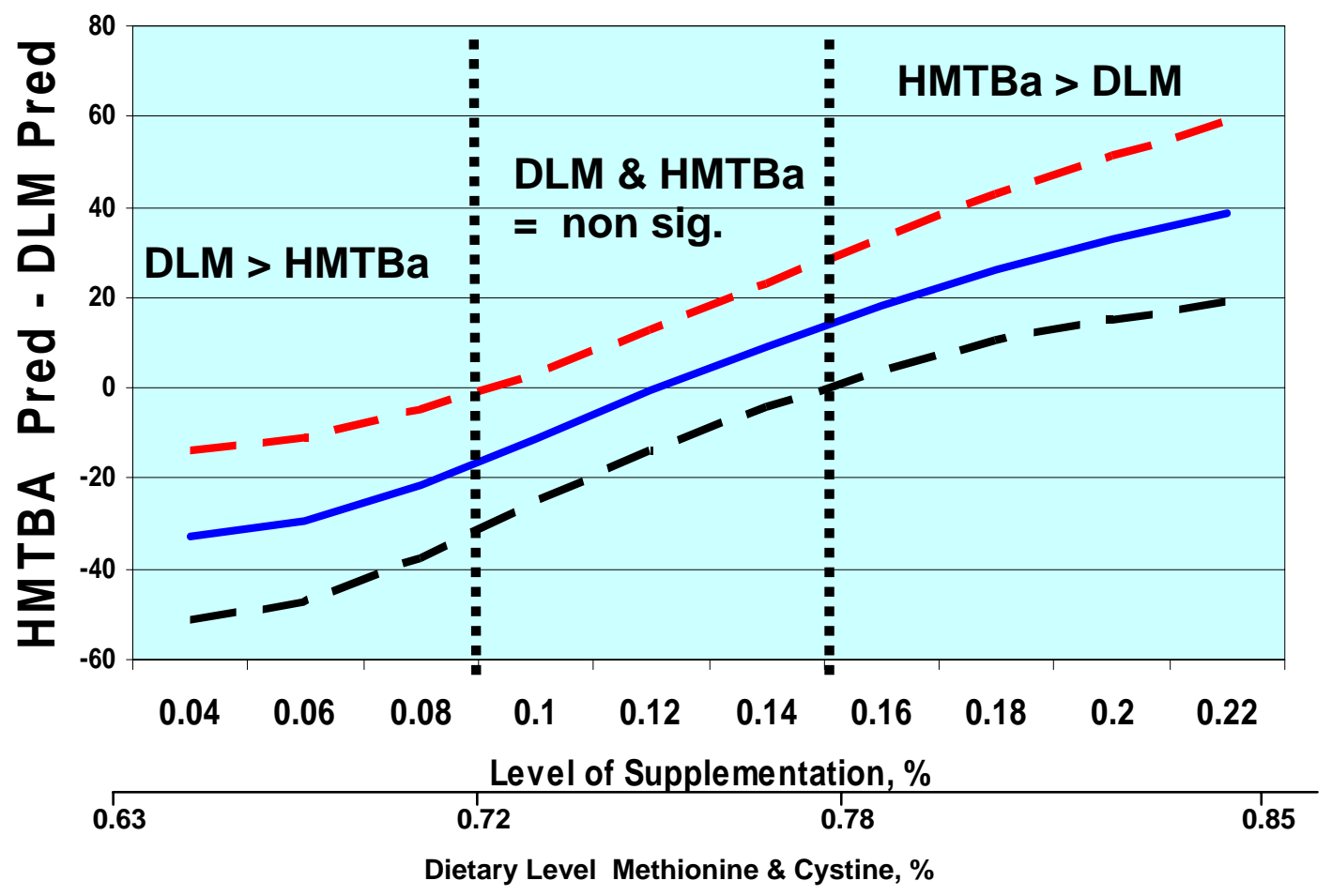

- Predicted Differences (HMTBA-DLM) - - Lower CL — - Upper CL 